\title{
La salud sexual y reproductiva de los estudiantes de nivel preparatoria: Un marco de análisis para la intervención de enfermería en la comunidad
}

\section{The sexual and reproductive health of high school students: A framework of analysis for community nursing intervention}

\author{
DOI: $10.46932 / \mathrm{sfjdv2n2-044}$
}

Received in: january 1st, 2020

Accepted in: March 30th, 2020

\section{Guillermina García Madrid \\ DCF}

Docente recién Jubilada de la Facultad de Enfermería de la Benemérita Universidad Autónoma de

Puebla.

guillergm25@ hotmail.com

\section{Maricarmen Moreno Tochihuitl}

Docente de la Facultad de Enfermería de la Benemérita Universidad Autónoma de Puebla.

María Laura Méndez Ovando

Docentes de la Facultad de Enfermería de la Benemérita Universidad Autónoma de Puebla

Jorge Antonio Ramos Vázquez

Docentes de la Facultad de Enfermería de la Benemérita Universidad Autónoma de Puebla

Gisela Ortiz Ramírez

Docentes de la Facultad de Enfermería de la Benemérita Universidad Autónoma de Puebla

\section{Alí de León Gómez}

Docentes de la Facultad de Enfermería de la Benemérita Universidad Autónoma de Puebla

Carmen Cruz Rivera

Docentes de la Facultad de Enfermería de la Benemérita Universidad Autónoma de Puebla

\section{Rayo Cruz Rivera}

Docentes de la Facultad de Enfermería de la Benemérita Universidad Autónoma de Puebla

\section{RESUMEN}

En el marco del Cuidado de la Salud Familiar patrocinado por el Consejo Internacional de Enfermería (CIE, 2002) y respaldado en México por el Programa "Escuela Saludable" de la Secretaría de Salud (S. S., 2010), el estudio del funcionamiento familiar, es un indicador elemental a realizar en las escuelas a los estudiantes, debido a su relación con el rendimiento escolar. Por ello, el objetivo del trabajo fue identificar cómo perciben el funcionamiento de sus familias los estudiantes de nivel preparatoria de una universidad pública de la ciudad de Puebla, México. Se trabajó con 216 estudiantes seleccionados aleatoriamente a quienes se les aplicó la Escala de Efectividad en el Funcionamiento Familiar de la Dra. Ma. Louise Friedemann. La edad de los estudiantes osciló entre los 13 y 18 años, las familias se caracterizaron porque el $71.4 \%$ son de tipo nuclear, el $40.8 \%$ tiene un promedio de 5 a 6 integrantes, el $60.6 \%$ es de una comunidad rural, el $80.6 \%$ es de un nivel socioeconómico medio y el $82 \%$ practica la religión católica. En relación a la percepción del funcionamiento familiar el $44.18 \%$ de estudiantes la percibe con un 
funcionamiento familiar alto, el $18.44 \%$ con un funcionamiento familiar medio y el $37.38 \%$ con un funcionamiento familiar bajo. La dimensión mantenimiento del sistema relacionada con las costumbres, creencias, hábitos, rutinas, reglas, normas y patrones de crianza tuvo la media más alta $52.29(D E=5.66)$ y la dimensión coherencia que engloba las relaciones familiares, la expresión de lazos de afecto, amor cariño, comunicación, apoyo y convivencia; factores que le dan un sentido de unidad, pertenencia y compromiso a la familia la media más baja $16.39(D E=5.27)$. Se concluye que la percepción del funcionamiento familiar depende de alguna manera de la etapa de vida de las personas encuestadas.

Palabras clave: funcionamiento familiar, estudiantes, nivel medio superior.

\begin{abstract}
Within the framework of Family Health Care sponsored by the International Council of Nursing (ICN, 2002) and supported in Mexico by the "Healthy School" Program of the Ministry of Health (S. S., 2010), the study of family functioning is an elementary indicator to be performed in schools to students, due to its relationship with school performance. Therefore, the objective of this study was to identify how high school students of a public university in the city of Puebla, Mexico, perceive the functioning of their families. We worked with 216 randomly selected students who were administered Dr. Ma. Louise Friedemann's Family Functioning Effectiveness Scale. The age of the students ranged between 13 and 18 years, the families were characterized by the fact that $71.4 \%$ are nuclear, $40.8 \%$ have an average of 5 to 6 members, $60.6 \%$ are from a rural community, $80.6 \%$ have a medium socioeconomic level and $82 \%$ practice the Catholic religion. In relation to the perception of family functioning, $44.18 \%$ of the students perceive it as high family functioning, $18.44 \%$ as medium family functioning and $37.38 \%$ as low family functioning. The dimension maintenance of the system related to customs, beliefs, habits, routines, rules, norms and patterns of upbringing had the highest mean 52.29 ( $\mathrm{SD}=5.66)$ and the dimension coherence that encompasses family relationships, the expression of bonds of affection, love, communication, support and coexistence; factors that give a sense of unity, belonging and commitment to the family had the lowest mean 16.39 ( $\mathrm{SD}=5.27)$. It is concluded that the perception of family functioning depends in some way on the life stage of the respondents.
\end{abstract}

Key words: family functioning, students, high school level.

\title{
1 INTRODUCCIÓN
}

La adolescencia y la juventud, son etapas que se caracterizan por la energía, vigor y frescura de los individuos. Si bien se trata de grupos de edad relativamente saludables son etapas que los exponen a múltiples factores de riesgo en el ejercicio de su sexualidad. La falta de información y educación sexual sobre medidas preventivas, sexo seguro y protegido, métodos anticonceptivos, uso y colocación del condón, infecciones de transmisión sexual y embarazo no deseado los llevan al desarrollo de comportamientos y prácticas inadecuadas, conflicto en la identidad de género, adquisición de hábitos dañinos, interacción con múltiples parejas sexuales y una toma de decisiones inadecuada respecto a su salud sexual, comprometiendo su bienestar presente y futuro (Organización Panamericana de la Salud, 2008).

Para la Organización Mundial de la Salud (OMS), 2006/2010), la salud sexual y reproductiva (SSR) es el estado de completo bienestar físico, mental y social, y no solamente la ausencia de enfermedad 
en todas las cuestiones relativas al aparato reproductor y sus funciones y procesos; componente esencial de la capacidad de los individuos para transformarse en personas equilibradas, responsables y productivas dentro de la sociedad, derecho que incluye el beneficio de la seguridad e integridad sexual, la identidad de género, la expresión y ejercicio de la sexualidad de manera segura, la privacidad, la igualdad, la expresión del amor y el acceso a la atención de la salud.

Garantizar la salud y los derechos sexuales y reproductivos de los adolescentes y jóvenes es fundamental para asegurar que tengan vidas más saludables. Por lo que el cuidado de la SSR debe darse a lo largo de todo el ciclo vital humano y en todos los contextos: familia, instituciones educativas y de salud, con el propósito de promover una buena toma de decisiones en el ejercicio de la sexualidad y reproducción, limitar las infecciones de transmisión sexual, los embarazos no deseados y los proyectos de vida truncados. Aspectos que deben ser preocupación central de los programas y profesionales de la salud (Bernstein y Hansen, 2006; Lerma et al, 2009).

En México, en el año 2012, los resultados de la Encuesta Nacional de Salud y Nutrición (ENSANUT) (Gutiérrez, Rivera et al, 2012) reportaron que el 23\% de adolescentes (de un total de 22804 083 millones; $50.3 \%$ hombres y $49.7 \%$ mujeres) tenía vida sexual activa, el $14.7 \%$ de los hombres y el $33.4 \%$ de las mujeres no utilizaron ningún método de protección en la primera relación sexual. Sin embargo, al momento de la encuesta el $80.6 \%$ manifestó utilizar el condón cuando tiene relaciones sexuales y el $6.2 \%$ de mujeres tomar hormonales, específicamente la píldora del segundo día. De las mujeres de 12 a 19 años de edad la mitad (51.9\%) refirió haber estado embarazada y el 10.7\% estaba cursando un embarazo al momento de la entrevista. En este mismo año el Instituto Nacional de Estadística y Geografía (INEGI, 2012) informó que las principales infecciones de transmisión sexual reportadas por las instituciones de salud en adolescentes y jóvenes fueron la candidiasis urogenital y el virus del papiloma humano (VPH), y el Instituto Mexicano del Seguro Social (IMSS) ${ }^{6}$ que el 32\% de la población afectada con VIH/SIDA tiene menos de 25 años, y la gran mayoría se contagió entre los 15 y 18 años de edad.

Respecto a los estudiantes universitarios poblanos la realidad antes mencionada no es diferente, los afectos, proyectos, emociones, hábitos y fantasías que forman parte de su vida durante su formación profesional, generan que algunos inicien su vida sexual activa a temprana edad y otros la continúen, la relación de pareja ocupa un lugar fundamental en sus intereses cotidianos, protagonizan noviazgos o relaciones amorosas fortuitas, junto con sus anhelos profesionales, sin embargo, la maternidad o paternidad no planeada y las infecciones de transmisión sexual, son un riesgo latente en ellos y generalmente se asocian a proyectos de vida truncados.

Ospina y Manrique (2007), al estudiar la SSR en 764 estudiantes universitarios encontraron que el $67.6 \%$ reconoce la existencia de otras preferencias sexuales, el 90.3\% calificó su educación sexual como 
adecuada, el 36.2\% consideró tener certeza absoluta de evitar un embarazo no deseado, el $66.2 \%$ consideraron necesario incrementar conocimientos sobre sexualidad para sentirse seguros, el $1.5 \%$ tienen parejas sexuales del mismo sexo, el $8.3 \%$ es bisexual; el promedio de edad inicio de vida sexual activa fue de 16.5 años, el promedio de compañeros sexuales fue de 3.96 en hombres y de 2 en mujeres. De igual manera Chávez, Petrzelová y Zapata (2009), reportaron que de 719 estudiantes universitarios mexicanos que participaron en su estudio sobre SSR, el 70\% recibió educación sexual de parte de la familia sobre ITS y métodos anticonceptivos, el 60\% conocía el uso del preservativo, el 51\% opinó que las relaciones entre homosexuales son anormales, el 13\% dijo que los homosexuales son enfermos y el $41 \%$ manifestó haber iniciado vida sexual activa entre $\operatorname{los} 18$ y 21 años. Por otro lado Lema et $\mathrm{al}^{9}$ encontraron que el $70.7 \%$ de 587 estudiantes universitarios eran heterosexuales, el 5.2\% homosexuales, el $33.6 \%$ consulta revistas, videos o páginas pornográficas y el $8.3 \%$ tiene relaciones sexuales virtuales. Finalmente González et al (2013), al estudiar la SSR en estudiantes universitarios en una comunidad Cubana hallaron que de 126 jóvenes que declararon haber tenido relaciones sexuales el 86.6\%, no utilizó ningún método de planificación familiar en su primera relación sexual; el 13.4\%, sí lo hizo y seleccionaron el condón, tabletas anticonceptivas y el coito interruptos.

En este contexto el presente estudio se dio a partir de la implementación del Programa "Cuidado de la Salud Sexual y Reproductiva de los Estudiantes Universitarios” (CUSASEX) (García, García M, 2013), donde los docentes del Departamento de Educación en Enfermería Comunitaria de la Facultad de Enfermería de la Benemérita Universidad Autónoma de Puebla, México, realizaron un diagnóstico previo sobre las problemáticas más comunes que comprometen la salud de los estudiantes de nivel preparatoria. El objetivo fue valorar conocimientos, conductas y prácticas sexuales y reproductivas de los estudiantes de tal manera que las intervenciones preventivas a implementar en la jornada "Cuida tu Salud" fueran asertivas y fortalecieran el programa "CUSASEX". Programa que desde el año 2006 se ha venido implementando año con año como una estrategia de prevención positiva en los estudiantes de las escuelas secundaria y telesecundaria del Centro Comunitario (CECACVI-BUAP) de San Andrés Azumiatla, en la Facultad de Enfermería, en preparatorias y otras Unidades Académicas de la BUAP y en instituciones gubernamentales de la ciudad de Puebla (Casa de la Juventud y escuelas secundarias y preparatorias de la Secretaría de Educación Pública de la ciudad de Atlixco y Puebla, México) en el cual participan profesionales de la salud de otras instituciones como la Secretaría de Salud del Estado de Puebla en especial El Centro Ambulatorio para la Prevención y Atención en SIDA e Infecciones de Transmisión Sexual (CAPASITS), el Instituto Mexicano del Seguro Social (Primer Nivel de Atención), el Hospital Universitario y la Facultad de Psicología de la BUAP., entre otros. Y cuyas acciones fortalecen al Cuerpo Académico de Enfermería Comunitaria. 


\section{METODOLOGÍA}

El estudio fue descriptivo, correlacional, transversal, realizado en 318 estudiantes de nivel preparatoria seleccionados al azahar a quienes en su salón de clase se les aplicó un instrumento de 36 preguntas sobre la salud sexual y reproductiva con un alpha de Crombach de .94, previa información del propósito del estudio y del manejo de la información. En la aplicación de los instrumentos participaron docentes integrantes del Departamento de Educación en Enfermería Comunitaria quienes mantuvieron la individualidad y seguridad de los entrevistados y preservaron el respeto a su dignidad y bienestar como lo marca el Reglamento de la Ley General de Salud en Materia de Investigación (Secretaría de Salud, 1984/2013). Para la captura y el análisis de los datos se utilizó el paquete estadístico SPSS (Statiscal Package for the Social Sciencies) versión 19.0, así como estadística descriptiva, de variabilidad y paramétrica de acuerdo a la curva de normalidad de los datos.

\section{RESULTADOS}

De la población encuestada, el promedio de edad fue de 17 años, el 54.2\% fueron mujeres y el 45.8\% hombres, el $83.7 \%$ eran solteros, el $87.0 \%$ recibió información sobre aspectos relacionados a la SSR, en su mayor porcentaje por profesionales de la salud en la escuela y padres de familia en el hogar (tabla 1/ gráfico 1), el $65.7 \%$ manifestó que la información recibida fue oportuna, veraz y suficiente, el $30.1 \%$ que fue oportuna y veraz pero insuficiente y el 3.3\% insuficiente e inadecuada.

Tabla 1. Recibió información y educación sobre salud sexual y reproductiva

\begin{tabular}{lcc}
\hline \multicolumn{1}{c}{ Respuesta } & $f$ & $\%$ \\
\hline SI & 303 & 87.0 \\
NO & 15 & 13.0 \\
\hline Fuente. ESSR. Puebla, México. 2012. & & 318 estudiantes
\end{tabular}

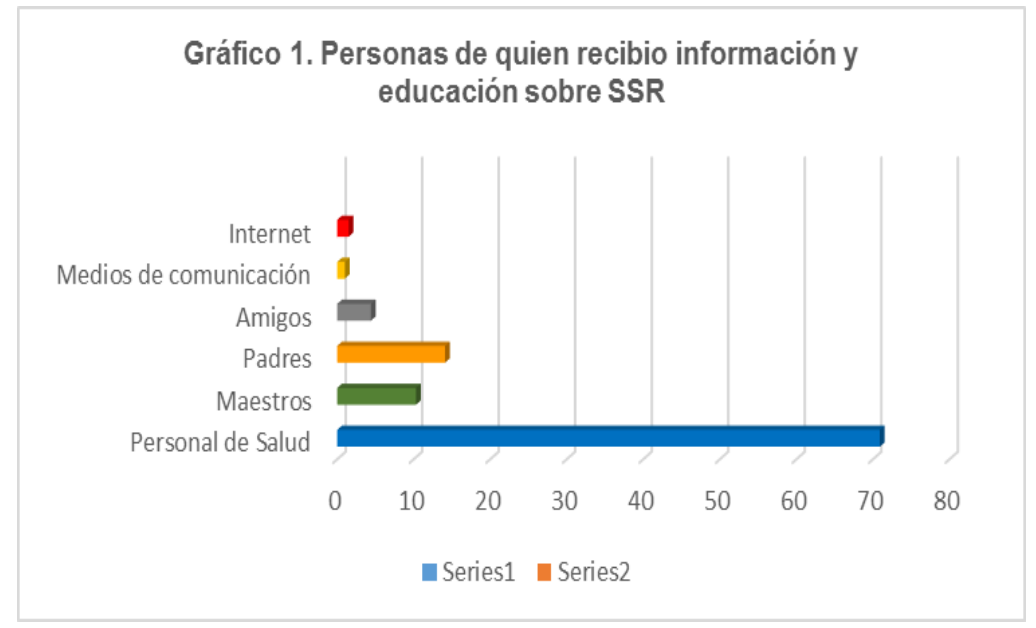

Fuente: ESSR. Puebla, México. 2012. 318 estudiantes 
El 91.5\% sabe cuáles son los factores de riesgo, que es una infección de transmisión sexual, para qué sirve el condón y cuál es la técnica correcta de su colocación. El 90.2 se identifica con su género (masculino - femenino), el 35.9\% tiene vida sexual activa (VSA), el 21.6\% la inicio de los 12 a los 13 años de edad y el 78.4\% entre los 14 y 17 años, el $40.8 \%$ con VSA práctica sexo seguro y protegido, el 92.4\% tiene una sola pareja sexual, el $6.9 \%$ de mujeres se ha embarazado de manera no planeada. No hubo relación de la edad y el género con la práctica de sexo seguro y protegido $(\mathbf{r}=.213, \mathbf{p}=.187 ; \mathbf{r}=$ $0.50, \mathbf{p}=.758)$, pero si con haber recibido información $(\mathrm{r}=.362, \mathrm{p}=.022)$.

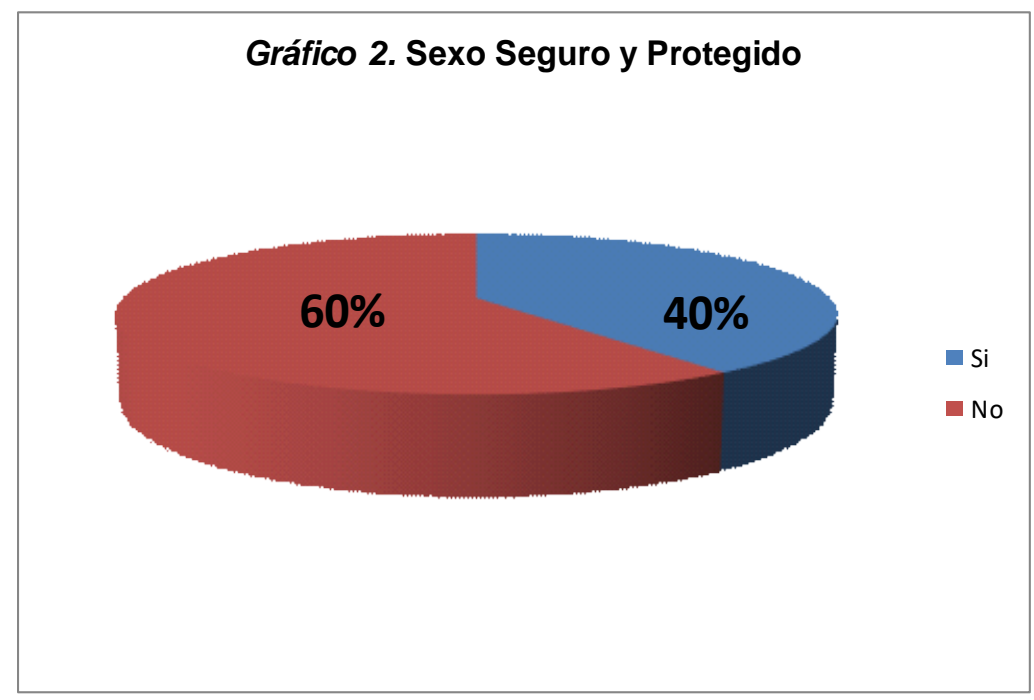

Fuente: ESSR. Puebla, México. 2012. 318 estudiantes

\section{DISCUSIÓN}

La implementación de programas de salud orientados a la educación y prevención de problemas de salud sexual y reproductiva como el programa "CUSASEX" en los estudiantes de nivel preparatoria, da como resultado que estos asuman con una mejor toma de decisiones y una mayor corresponsabilidad el ejercicio de su sexualidad y de sus comportamientos y prácticas sexuales.

Los datos obtenidos en el estudio, de manera general coinciden con los obtenidos en otras escuelas y facultades en donde se ha implementado el programa, muestran un desarrollo de conductas y prácticas sexuales de riesgo bajo. La edad de inicio de la VSA coincide con lo reportado por el Instituto Nacional de Estadística y Geografía en estudios realizados en adolescentes, así como en la Encuesta Nacional de Nutrición y Ospina y Manrique, sin embargo, difieren con los de Chávez, Petrzelová y Zapata quienes encontraron que la edad de inicio fue de los 18 a los 21 años, es decir a una edad mayor. Respecto a la información y educación recibida los resultados muestran semejanza con los publicados por Chávez, Petrzelová y Zapata quienes informan que un alto porcentaje de adolescentes manifestó tener una 
educación sexual adecuada y haber recibido información sobre diversas temáticas de SSR, en especial sobre el uso del condón, no concuerdan con la información reportada por Ospina y Manrique en donde los estudiantes consideraron necesario incrementar conocimientos sobre sexualidad para sentirse más seguros. Los resultados obtenidos evidenciaron una alta exposición al riesgo de enfermedades de transmisión sexual y embarazos no deseados de parte de los estudiantes, por lo que se implementaron intervenciones dirigidas a limitar el riesgo, fortalecer el sexo seguro y protegido, la corresponsabilidad de los estudiantes en el cuidado de su salud sexual y reproductiva.

Finalmente la escuela como institución mediadora entre la dimensión social y la individual busca garantizar una formación integral en los estudiantes y limitar cualquier factor de riesgo a la salud que trunque los proyectos de vida de los mismos. 


\section{REFERENCIAS}

1. Bernstein, S. y J. Hansen. (2006). “Opciones públicas, decisiones privadas: Salud Sexual y reproductiva y los Objetivos de Desarrollo del Milenio". Nueva York, Estados Unidos: Programa de las Naciones Unidas para el Desarrollo. Recuperado en: http://www.generoysaludreproductiva.gob.mx/IMG/pdf/Opcionespublicasdecisionesprivadas-2.pdf

2. Chávez, M., Petrzelová, J. y Zapata, J. (2009). Actitudes respecto a la sexualidad en estudiantes universitarios. Enseñanza e Investigación en Psicología. 2009. 1 (14), pp.137-151

3. González, P. L.; González, L. N.; Mena, F. M.; Navarro P. A. y Martín L. X. (2013). Salud Sexual y Reproductiva en Estudiantes Universitarios. ¿Modo y Estilo de Vida Saludables?, Un diagnóstico. Instituto Superior de Ciencias Médicas Carlos J. Finlay Camaguey, Cuba. Rev Hum Med [online]. Vol.3, n.1, pp.

4. García R. R.; García M. G.; Moreno, T.MC. (2013). Programa "Cuidad tu Salud: Cuidado de la Salud Sexual y Reproductiva de los Estudiantes Universitarios" Facultad de Enfermería de la Benemérita Universidad Autónoma de Puebla, Departamento de Educación en Enfermería Comunitaria. México.

5. Gutiérrez, J. P.; Rivera, D. J.; Shamah, L. T.; Villalpando, H. S.; Franco A, Cuevas, N. L.; Romero, M. M. y Hernández, Á. M. Encuesta Nacional de Salud y Nutrición 2012. Resultados Nacionales. Cuernavaca, México: Instituto Nacional de Salud Pública (MX), 2012.

6. Instituto Nacional de Estadística y Geografía (INEGI). Mujeres y hombres en México 2012. México. 2013.

7. Instituto Mexicano del Seguro Social. (2010). En México, 30\% de jóvenes ha contraído alguna enfermedad sexual. Milenio, México. D. F. Recuperado en: http://sipse.com/archivo/en-mexico-30-dejovenes-ha-contraido-alguna-enfermedad-sexual-28293.html

8. Lema, S. L., Salazar T. I., Varela, A. M., Tamayo, C. J., Rubio S. A. y Botero, P. A. Comportamiento y salud de los jóvenes universitarios: satisfacción con el estilo de vida. Pensamiento Psicológico. 2009. 5 (2), pp. 71-87.

9. Organización Panamericana de la Salud. (2008). La Salud Sexual y Reproductiva de los Adolescentes y los Jóvenes: Oportunidades, Enfoques y Opiniones. Washington, D. C. OPS, ()

10. Organización Mundial de la Salud (OMS). (2001/2010). La salud sexual y su relación con la salud reproductiva: un enfoque operativo. Recuperado https://apps.who.int/iris/bitstream/handle/10665/274656/9789243512884-spa.pdf

11. Ospina, D. J. M, Manrique, A. F. G. (2007). Prácticas y Comportamientos Sexuales en Estudiantes Universitarios. Av. Enferm. 25(2): 101-111.

12. Secretaría de Salud. (1984/2013). Ley General de Salud: Reglamento en Materia de Investigación para la Salud. Recuperado en: http://www.salud.gob.mx/unidades/ 